\title{
Saxagliptin Hydrochloride
}

National Cancer Institute

\section{Source}

National Cancer Institute. Saxagliptin Hydrochloride. NCI Thesaurus. Code C81702.

The hydrochloride salt form of saxag liptin, an orally bioavailable, potent, selective and competitive, cyanopyrrolidine-based inhibitor of dipeptidyl peptidase 4 (DPP-4), with hypoglycemic activity. Saxagliptin is metabolized into an, although less potent, active mono-hydroxy metabolite. 